\title{
Metric analysis of the hard palate in children with Down syndrome - a comparative study
}

\section{Gopalan Bhagyalakshmi', Annappa Jai Renukarya ${ }^{2}$ and Sayee Rajangam ${ }^{3}$}

The hard palate is viewed as playing an important role in the passive articulation of speech. Its probable role in the defective articulation of speech in individuals with Down syndrome has been examined in the present study. In individuals with Down syndrome, the hard palate is highly arched, constricted, and narrow and stair type with malformed misaligned teeth and a large and fissured tongue. As a result good palato-lingual contact is not achieved, with resulting defective articulation. Using orthodontic and prosthodontic principles could modify this situation, i.e. the anatomy of the hard palate. The altered palatal contour may give better placing to the tongue, leading to improved palato-lingual contact and articulation. The dimensional parameters measured were: average linear width (AVL), average curvilinear width (AVCL), average height $(\mathrm{AVH})$ at different planes; average antero-posterior length (AAP), average volume $(V)$, palatal arch length (PAL), and palatal index (PI). The findings were compared with those of controls of the same age and sex. The AVL, AVCL, AAP, PAL, V and PI values of patients with Down syndrome were found to be less than the corresponding values of controls and the average height values of patients with Down syndrome were greater than the corresponding values of controls. Statistical significance was observed in all measurements between the controls and the patients with Down syndrome, especially in those concerning the height and the volume of the oral cavity. Observations from this study have suggested that prostheses might be designed to modify the palatal anatomy and produce better articulation in people with Down syndrome.

(1) Department of Anatomy, R.V. Dental College, J.P. Nagar, Bangalore

(2) Department of Anatomy, Bangalore Medical College, Bangalore

(3) Division of Human Genetics, St John's Medical College, Bangalore

Correspondence to Dr. G. Bhagyalakshmi • e-mail: sjmcdhg@yahoo.com

doi:10.3104/reports.1999

(๑) 2007 The Authors. Journal Compilation @ 2007 The Down Syndrome Educational Trust.
The hard palate is a neglected area in the field of anatomical research as there is little morphological or metric analysis carried out in this area. The hard palate in the present study has been viewed only as an important passive aid to speech and as a probable contributing factor in the defective articulation of speech in individuals with Down syndrome.

Textbooks describe the hard palate in individuals with Down syndrome as high arched, constricted, narrow and stair type. These findings are based on clinical examination. The characteristic teeth in individuals with Down syndrome and the bald and fissured tongue may be treated conservatively but not the hard palate and relatively large tongue. Given this situation, it has been thought that the anatomy of the hard palate might be modified to an extent by applying orthodontic and prosthodontic principles. In turn, the palatal contour and volume might be modified, leading to the better placing of the tongue inside the oral cavity; this might lead to improved palato-lingual contact and hence improved articulation of speech. Based on this view, this study has identified certain dimensional parameters of the hard palate and measured these with impression models in children with Down syndrome and in a control group of typically developing children.

\section{Aims and objectives}

- To record a detailed history of the patients.

- To carry out general physical examination of the oral cavity and also the relevant systemic examination.

- To obtain dental palatal impressions of the selected participants, typically developing and with Down syndrome.

- To identify the parameters to be measured.

- To measure these parameters on the impression models of children with Down syndrome and typically developing children

- To carry out a comparative analysis and draw the statistical significance. 


\section{Methods}

Eighty-eight children with Down syndrome aged from 6 to 16 years took part (46 males and 42 females). Forty-eight children (26 males and 22 females) underwent examination as well as having impressions taken of their maxillary teeth and hard palate. The number of controls was also 48 (males 26, females 22). Electronic calipers, scale, divider, Civil L, dental impression material, impression trays, plaster stone, casting tray, modelling wax, file, coconut oil, $5 \mathrm{cc}$ syringe and refined clay were the items used.

Dental impressions of the 48 children with Down syndrome and the 48 controls were taken with the help of dentists. After drying and casting, the impressions were measured (FIGURE 1).

The parameters measured were:

- Mean linear width (AVL)

- Mean curvilinear width (AVCL)

- Mean height (AVH)

- Mean palatal arch length (PAL)

- Mean antero-posterior length (AAP)

- Mean volume (V)

- Palatal index (PI) - Max. Width / length x 100

Measuring AVL at A, a, b, c, d and e: Using electronic calipers, the tips of the mesial gingival papillae between the teeth on either side at $A, a$, b, c, d and e planes were measured in $\mathrm{cms}$. The procedure was carried out twice. An average of the two measurements at planes A, a, b, c, d and e was recorded as the AVL.

Measuring AVCL and AVH at A, a, b, c, d and e: Strips of modelling wax were fastened along the roof of the palate along the planes $A, a, b, c$, $\mathrm{d}$ and e between the tips of the gingival papillae across the width of the palate. Later these strips were removed carefully from the roof of the palate with the arch intact and transferred on to a sheet from where the parabola was obtained. The ends of the curvature were inverted over a straight-line base touching both ends of the parabola. The highest point on the parabola was marked and a perpendicular was dropped to the straight-line base. This method gave the palatal heights $(\mathrm{AVH})$ corresponding to the planes $\mathrm{A}$, a, $b, c, d$ and $e$, in the median plane. The procedure was carried out twice. The average of the two readings was recorded as $\mathrm{AVH}$ at different planes in cms. After measuring the heights the curved strips were straightened and, with the help of a scale, the curvilinear widths corresponding to A, $a, b, c, d$ and e planes were measured. The procedure was repeated and the average of the two readings was recorded as the AVCL at a, b, c, d and e planes in cms.

Measuring PAL (Palatal Arch Length): A thread was taken, wetted, and its end was applied to the a.

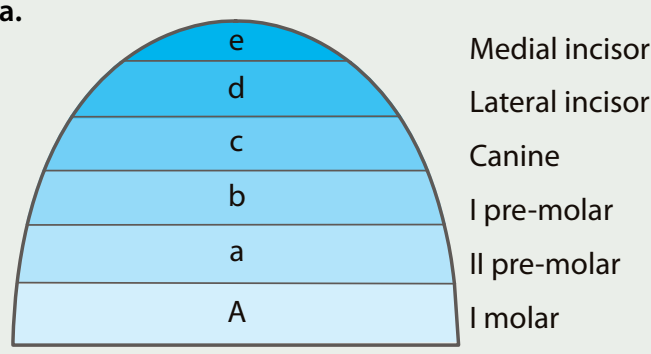

Planes used in metric analyis of the hard palate

b.

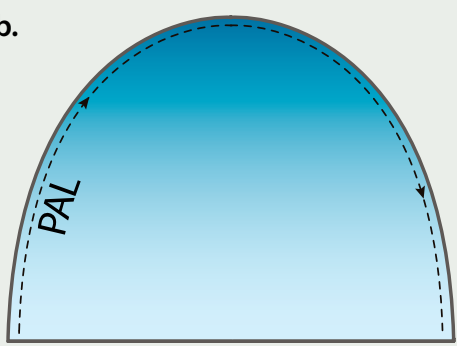

c.

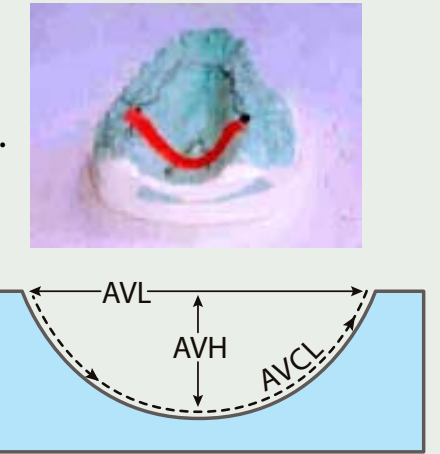

d.
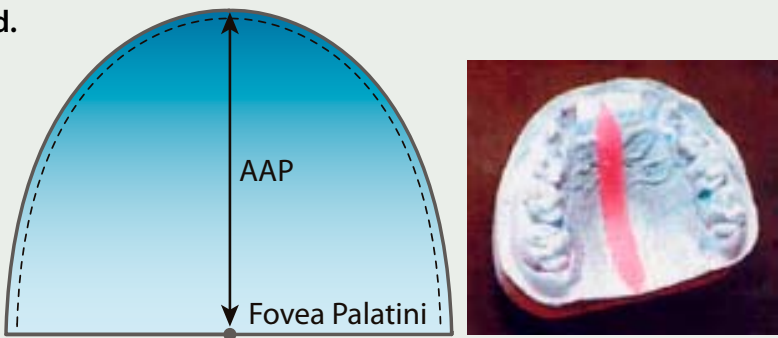

Figure 1 | Parameters measured from the impressions. a | Planes used in metric analysis. b | Palatal arch length (PAL). c | Average linear width (AVL) average curvilinear width (AVCL). d $\mid$ Average antero-posterior length (AAP).

tip of the posterior-most gingival papilla at one end of the model and taken to the end of the tip of the posterior-most gingival papilla at the other end, touching the tips of all the intervening gingival papillae across the impression model. The procedure was repeated and the average of the two readings was recorded as the AAP length in cms.

Measuring AAP (Average Antero Posterior Length): The tip of the most anterior gingival papilla in the median plane was located and, with the help of the strip of modelling wax, the anteroposterior distance between the point mentioned above and a point at the fovea palatini was measured along the median antero-posterior plane of the model. The procedure was carried out twice and the average of the two readings was recorded as the AAP in cms.

Measuring (V) volume: The impression models were soaked in water overnight to prevent absorption of water due to the hygroscopic nature of the impression material and were then dried. Coconut oil was lightly smeared over the surface of the vault of the impression models of study and control groups. The posterior gap of the palatal vault between the posterior most molars was then bridged with refined clay, the bridge extend- 
ing along the palatal foveae in the midline and stretching across the vault on either side and to a height corresponding to a plane connecting tips of all gingival papillae across the model. The wall surface towards the vault was well levelled with Civil L. Water was then poured into the palatal dome so as to bring the water level to just cover the tips of the gingival papillae across the palatal vault. The procedure was carried out twice and the average of the two volume readings was taken as the average volume $(\mathrm{V})$ in $\mathrm{ml}$.

PI (Palatal index calculation): This was calculated using the formula: Maximum width of the palatal arch x 100/ Maximum length of the palatal arch (PAL). The exercise was carried out with the readings of controls and the study group provided by the highest parameters of the AVL and PAL.

All 22 parameters of both the study group and control group were tabulated, and means and standard deviation were calculated. The student ' $t$ test' and standard deviation values of the study and the control groups were calculated.

An alternative method was also proposed to detect the depth of the palatal dome. A well-circumscribed highest plane of an inverted $U$ shape was marked in the vault of the impression models of study and control groups. Five points were marked at equidistance: two points L1 and L2 on the left side, two points R1, R2 on the right side, and a middle point $\mathrm{M}$ was marked in the middle along the median plane on the $\mathrm{U}$ shaped plane.

Another lower plane including the tips of all the gingival papillae of the shape naturally being an inverted $U$ correlating to the alveolar margins of the maxillary teeth was identified. Five points were marked on this arc similar to the method mentioned above in the impression models of both study and control groups. An electronic caliper was placed vertically at each point such that the points R1, R2, M, L1 and L2 of both the planes were in a straight line vertically. This exercise was carried out on the study and control models on a flat surface in a well-lit room and at a level corresponding to that of the eye level of the person carrying out the exercise with the conditions mentioned above. The distances between the corresponding points on the lower plane and upper plane were measured by adjusting the screws of the calipers. The readings were recorded as heights at R1, R2, M, L1 and L2. This exercise was repeated again and the average of the two was recorded for gauging the height of the palatal vault. The readings for the study group and control group were tabulated and the ' $t$ test' was carried out on the data.

\section{Results}

TABLES 1 TO 5 show the mean and $S^{D}$ values for each parameter (one each for AVL, AVCL, AVH, Depth and one for AAP, PAL, V and PI); the graphic representations for the same values are also shown.

- AVL mean values of the study group were observed to be less than the mean values of the controls and the results were found to be statistically significant.

- AVCL mean values of the study group were observed to be less than the mean values of the controls and were found to be statistically significant.

- AVH mean values of the study group were observed to be greater than the mean values of the controls and these were found to be statistically significant.

- AAP, V, PAL and PI mean values of the study group were observed to be less than the mean values of the controls and were found to be statistically significant.

- Mean values of depths at different points on the hard palates of the study group were observed to be greater than the values of the controls.

\section{Review and discussion}

The literature on palatography has suggested its use as a means of demonstrating palato-lingual contact during articulation. Calculation of the Palatal Index (PI) by the formula PI = Palatal Height / Palatal Length has been mentioned, but methods of measuring these indices have not been described ${ }^{[1]}$. The successful use of oral-myofunctional therapy techniques in treatment of speech articulation disorders by palatal augmentation has been reported ${ }^{[2]}$. Results after assessment of adaptation to palatal modifications have been found to be $\operatorname{good}^{[3]}$. Three types of palatal plates have been designed to achieve different effects and $65-75 \%$ results have been achieved in all the three categories.

In a rehabilitative study, 25 patients with dysarthria of various types due to motor problems have been treated with palatal lift augmentation prostheses. Twenty-one patients showed improvement in their dysarthria, $10 \%$ had reduced hyper nasality and a $6 \%$ improvement in speech $^{[4]}$. Treating 69 children with Down syndrome by Castillo-Morale's therapy has resulted in improvement in tongue position, upper and lower lip tonicity and position, mouth closure, drooling and suckling ${ }^{[5]}$. Carlstedt et al., ${ }^{[6]}$ have reported palatal plate therapy as a valuable component to a training programme for improving oro-facial muscle function in children with 


\begin{tabular}{|lrrrrr|}
\hline & Study Group $(\mathbf{n}=\mathbf{4 8})$ & \multicolumn{3}{c|}{ Control $(\mathbf{n}=\mathbf{4 8})$} \\
\hline AVL $(\mathrm{cm})$ & Mean & SD & Mean & SD & $p$ value \\
A & 3.53 & 0.25 & 3.99 & 0.33 & $p<0.01$ \\
a & 3.31 & 0.24 & 3.77 & 0.19 & $p<0.01$ \\
b & 3.16 & 0.22 & 3.73 & 0.29 & $p<0.01$ \\
c & 2.95 & 0.26 & 3.34 & 0.28 & $p<0.01$ \\
d & 2.00 & 0.35 & 2.47 & 0.32 & $p<0.01$ \\
e & 1.30 & 0.27 & 1.48 & 0.30 & NS
\end{tabular}

Table 1 | AVL-Comparison of means and SD for study and control groups at different planes

\begin{tabular}{|lrrrrr|}
\hline & Study Group $(\mathbf{n}=\mathbf{4 8})$ & \multicolumn{3}{c|}{ Control $(\mathbf{n}=\mathbf{4 8})$} \\
\hline AVCL $(\mathrm{cm})$ & Mean & SD & Mean & SD & $p$ value \\
A & 4.28 & 0.41 & 4.70 & 0.44 & $p<0.05$ \\
a & 4.56 & 0.39 & 4.76 & 0.38 & NS \\
b & 4.15 & 0.47 & 4.48 & 0.52 & NS \\
c & 2.89 & 0.30 & 3.46 & 0.40 & $p<0.01$ \\
d & 2.179 & 0.27 & 2.51 & 0.33 & $p<0.01$ \\
e & 1.319 & 0.25 & 1.56 & 0.34 & $p<0.05$
\end{tabular}

Table 2 | AVCL-Comparison of means and SD for study and control groups at different planes

\begin{tabular}{|lrrrrr|}
\hline & Study Group $(\mathbf{n}=\mathbf{4 8})$ & \multicolumn{3}{c|}{ Control $(\mathbf{n}=\mathbf{4 8})$} \\
\hline AVH $(\mathrm{cm})$ & Mean & SD & Mean & SD & $p$ value \\
A & 1.20 & 0.44 & 1.06 & 0.20 & NS \\
a & 1.49 & 0.23 & 1.27 & 0.27 & $p<0.05$ \\
b & 1.63 & 0.35 & 1.48 & 0.32 & $p<0.05$ \\
c & 0.65 & 0.20 & 0.51 & 0.20 & NS \\
d & 0.44 & 0.19 & 0.33 & 0.22 & NS \\
e & 0.13 & 0.22 & 0.20 & 0.24 & NS
\end{tabular}

Table 3 | AVH-Comparison of means and SD for study and control groups at different planes

\begin{tabular}{|lrrrrr|}
\hline & Study Group $(\mathbf{n}=\mathbf{4 8})$ & \multicolumn{4}{c|}{ Control $(\mathbf{n}=\mathbf{4 8})$} \\
\hline & Mean & SD & Mean & SD & $p$ value \\
AAP $(\mathrm{cm})$ & 4.18 & 0.34 & 4.39 & 0.39 & NS \\
V $(\mathrm{ml})$ & 4.27 & 0.90 & 6.41 & 1.68 & $p<0.01$ \\
PAL $(\mathrm{cm})$ & 7.83 & 0.84 & 8.66 & 0.89 & $p<0.05$ \\
PI & 84.40 & 8.13 & 93.22 & 8.56 & $p<0.01$ \\
Table 4 | AAP, V, PAL, PI-Comparison of means and SD for \\
study and control groups at different planes \\
\hline
\end{tabular}

\begin{tabular}{|lcrrrr|}
\hline \multicolumn{7}{c|}{ Study Group ( $\mathbf{n}=\mathbf{4 8})$} & \multicolumn{3}{c|}{ Control $(\mathbf{n}=\mathbf{4 8})$} \\
\hline L1 & Mean & SD & Mean & SD & $p$ VALUE \\
L2 & 12.02 & 1.46 & 10.36 & 1.98 & $p<0.05$ \\
M & 13.38 & 1.45 & 11.6 & 1.99 & $p<0.01$ \\
R1 & 12.78 & 1.86 & 11.17 & 1.52 & $p<0.05$ \\
R2 & 12.06 & 1.54 & 10.23 & 1.96 & $p<0.01$ \\
Table 5 | Comparison of means and SD for study and \\
control groups at different points on the vault of the \\
hard palate
\end{tabular}

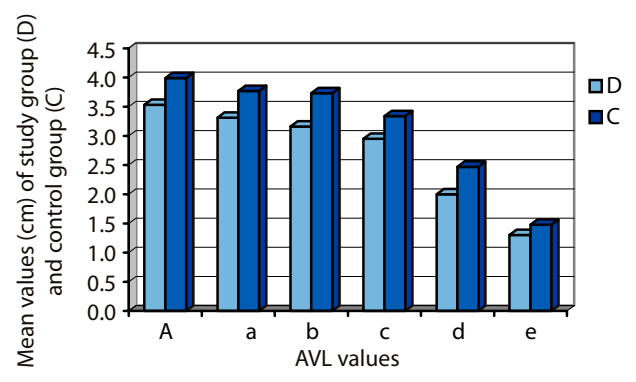

Figure 2 | Comparison of mean AVL values for study group and control group

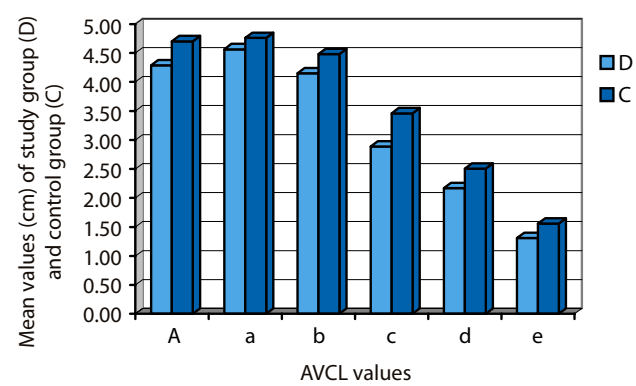

Figure 3 | Comparison of AVCL mean values for study group and control group at different planes

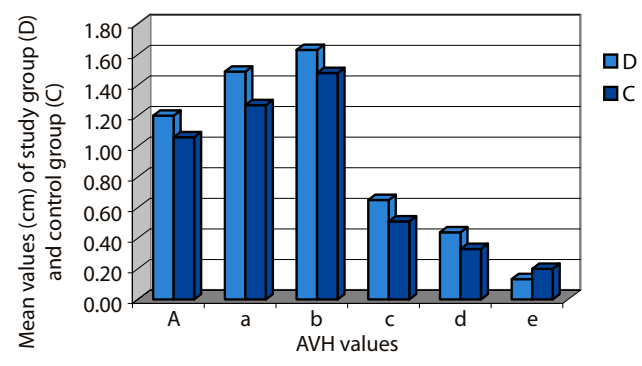

Figure 4 | Comparison of mean AVH values for study group and control group.

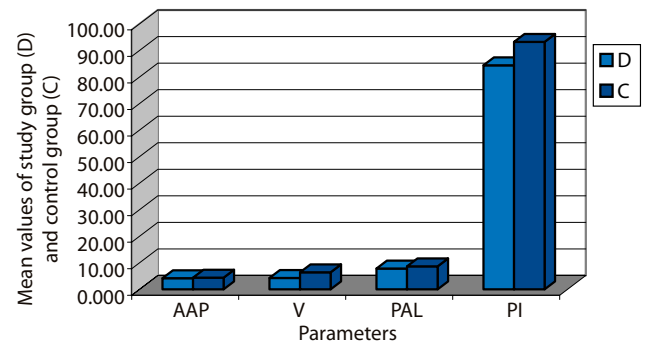

Figure 5 | Comparison of mean AAP, V, PAL and PI values for study group and control group

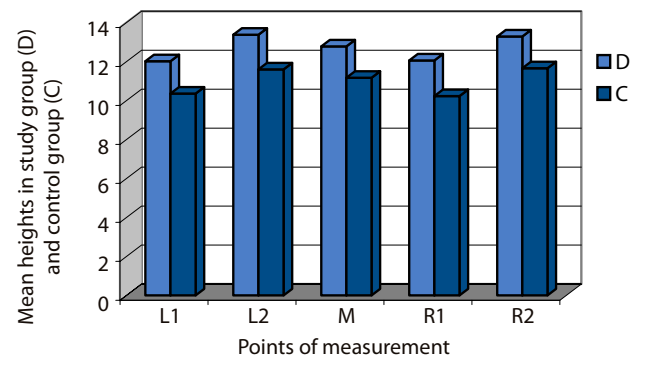

Figure 6 | Comparison of mean depth at different points for study group and control group. 
Down syndrome and also for better speech development ${ }^{[7]}$.

Orthodontic treatments of various types in children with Down syndrome with malocclusion have been described and encouraging results have been reported ${ }^{[8]}$. No studies in the field of palatometry were found. The present study has identified parameters from statistically significant differences in hard palate dimensions between children with Down syndrome and a control group. The hard palate in children with Down syndrome was found to be high arched and narrow, with acutely aligned palatine plates. The increase in palatal height is associated with a constricted palate, contributing to the projecting of the tongue in individuals with Down syndrome. During articulation, this factor could result in inefficient palato-lingual contact, resulting in defective phonation in these children. In addition, the angulation of the palatal plates of the maxillary processes may be acute during development, resulting in the constricted hard palate and smaller volume of the oral cavity.

The anatomically distorted hard palate could be modified to a great extent by means of expanding the palatal arch, a routine procedure in orthodontics.

The present study has provided a base-line in the measurement of dimensional parameters of the hard palate in children with Down syndrome and an age- and sex-matched control group. Appropriate strategies to improve palatal morphology in people with Down syndrome may be designed to improve their communication.
1. Howell S. Assessment of palatal height in children. Community Dentistry and Oral Epidemiology. 1981;9(1):44-47.

2. Landis CF. Applications of oro-facial functional techniques to speech therapy. International Journal of Orofacial Myology. 1994;20:40-51.

3. Baum SR, McFarland DH. Speech adaptation to an artificial palate. Journal of the Acoustical Society of America. 1997;102(4):2353-2359.

4. Esposito SJ, Mitsumoto H, Shanks M. Use of palatal lift and palatal augmentation prosthesis to improve dysarthria in patients with amyotrophic lateral sclerosis, a case series. Journal of Prosthetic Dentistry. 2000;83(1):90-98.
5. Limbrock GJ, Grandies F, Avalle C. CastilloMorales' Oro-facial therapy: treatment of 67 children with Down syndrome. Developmental Medicine and Child Neurology. 1991;339(4):296303.

6. Carlstedt K, Dahllof G, Nilsson B, Modeer T. Effect of palatal plate therapy in children with Down syndrome, a 1 year study. Acta Odontologica Scandinavica. 1996;54(2):122-125.

7. Hohoff A, Ehmer U. Effect of the Castillo-Morales stimulating plate on speech development of children with Down syndrome. A retrospective study. Journal of Orofacial Orthopaedics. 1997; 58(6):330-339.
8. Desai S, Flanagan J. Orthodontic considerations in individuals with Down syndrome: A case report. The Angle Orthodontist. 1999; Chris Burke: 85-88.

Received: 17 February 2004; Accepted 14 February 2007; Published online: 30 July 2007. 